\title{
The North Carolina Lactation Educator Training Program
}

Training Healthcare Professionals to Support Breastfeeding Women

Share this:

Georganna Cogburn, MS, RD, LDN, IBCLC, RLC ${ }^{1}$

Mona Brown Ketner, MSN, RN, C-EFM ${ }^{2}$

The North Carolina Lactation Educator Training Program (NCLETP) provides healthcare professionals working in health departments, hospitals, and care provider offices with the tools to educate women about breastfeeding and to support breastfeeding women to achieve their breastfeeding goals. In addition to 5 didactic days and an independent study, the NCLETP offers 2 clinical days, which gives learners an opportunity to apply the information learned in the classroom to clinical practice. The program empowers learners to return to their home communities and make a difference by providing breastfeeding education and support.

Keywords: breastfeeding education; lactation; staff; interdisciplinary

The American Academy of Pediatrics; the Academy of Nutrition and Dietetics; the United States Department of Health and Human Services; and the Association of Women's Health, Obstetric and Neonatal Nurses all recommend breastfeeding as the optimal form of infant nutrition. Furthermore, exclusive breastfeeding is recommended for the first 6 months of the infant's life.

The World Health Organization, Baby-Friendly Hospital Initiative, The Surgeon General's Call to Action, the American Academy of Pediatrics, and the Affordable Care Act all require that healthcare providers, including physicians, nurses, dietitians, counselors, and nursing assistants, be trained in basic breastfeeding management and be able to assist women with breastfeeding. Research shows that women who receive breastfeeding support from their healthcare provider are more likely to initiate breastfeeding and have longer breastfeeding durations. Healthcare providers are responsible for assuring that the information shared with patients is accurate and consistent. It has been identified that healthcare providers lack the lactation training necessary to assist women with breastfeeding issues and concerns. The ability to problem-solve breastfeeding concerns has been identified as an area where healthcare providers need more training (Watkins \& Dodgson, 2010).

A factor guiding the development of the North Carolina Lactation Educator Training Program (NCLETP) was the North Carolina breastfeeding rate. In the early 1990s, the North Carolina breastfeeding rates for all women were $55 \%$. In contrast, the U.S. rate was $71 \%$. The Healthy People goals for 2000 recommended that

1. georganna.cogburn@mahec.net

2.mketner@wakehealth.edu
$75 \%$ of women initiate breastfeeding. Because of the Surgeon General's recommendation for breastfeeding through the first year of life, and in the interest of women's and children's health, a group of interested professionals in North Carolina began a program to address this challenge.

In the mid-1990s, two IBCLCs, a public health educator, and a neonatologist began to brainstorm their vision for a statewide program to train hospital and health department staff members. Their goal was to support breastfeeding women across the entire state in a consistent and standardized manner.

The stated purpose was to meet the federal training requirements for breastfeeding education for staff of the North Carolina Special Supplemental Nutrition Program for Women, Infants, and Children (WIC) program. The planning group formulated an educational goal: to meet the knowledge gaps regarding lactation evidence-based practice guidelines for healthcare providers throughout the state. After much planning, the NCLETP officially started in May 1996.

\section{Program Implementation}

The NCLETP requires the collaboration of many partners: Northwest Area Health Education Center (AHEC) at Wake Forest School of Medicine, Novant Health Forsyth Medical Center, Forsyth County Department of Public Health WIC program, and the North Carolina Department of Health and Human Services. These partnerships have been essential for program implementation and continuation. Program faculty members represent many disciplines: IBCLCs, a neonatologist, registered nurses, a nurse practitioner, registered dietitians, and pediatricians. 
North Carolina has 100 counties and 87 WIC programs across state. Per federal requirements, all WIC programs are required to have a breastfeeding coordinator. Also, 81 of the WIC programs have a breastfeeding peer counselor program and have a breastfeeding peer counselor program manager. All of these WIC staff members are initially educated through the NCLETP. When there is a change of staff in the roles, the new person must be trained through the NCLETP within 12 months of assuming the new role. The local agency's breastfeeding coordinator and breastfeeding peer counselor program manager attend the NCLETP at least every 10 years to remain current in their breastfeeding knowledge. In addition, they must accrue 20 hours of breastfeeding continuing education every 5 years.

The NCLETP consists of 7 learning days: 3 initial classroom days, followed by 2 clinical days to apply classroom material, and then 2 additional didactic days. Participants receive learning materials, two reference books, and a tote bag imprinted with the NCLETP logo. An independent study is also included. The course content for the didactic days follows the International Board of Lactation Consultant Examiners (IBLCE) exam blueprint, which assures that the course is meeting the standards for the training of lactation consultants set forth by the IBLCE. It includes topics such as anatomy/physiology, counseling skills, ethics, medical lactation therapy, community support, current research, and cultural aspects. Other agenda items are breast pain, jaundice, special populations, troubleshooting, and workplace support for the breastfeeding mom. Employers expect that staff members attending this specialized training are able to use the material learned in the clinical days and apply critical thinking skills immediately after the training. The learners have requested more patient contact to apply the knowledge learned (Academy of Breastfeeding Medicine, 2011; Howett \& Lauwers, 2013; Maskey, 2008).

Learning strategies employed in the classroom days include didactic presentations, discussions, and roleplays. Others include question/answer sessions, videos, equipment demonstrations, and gaming. Learners actively participate in all sessions, with interdisciplinary education being one of the educational goals.

The two 6-hour clinical days are important aspects of this unique program. One day for program participants is spent at Novant Health Forsyth Medical Center, where the learners are able to be a part of neonatal intensive care unit rounds, work with IBCLCs on the inpatient mother-baby unit, and observe the IBCLCs counseling mothers on an outpatient basis. The second clinical day takes place at the Forsyth County Department of Public Health WIC program where learners participate in breastfeeding classes held in both English and Spanish, observe a WIC program in action, watch peer counselors in their roles, interact with mothers having breastfeeding concerns, and observe the use of breast pumps.

The independent study component of the NCLETP includes a personalized community breastfeeding assessment, a World Breastfeeding Week activity or hospital profile, competency questions, reference review, and evaluation case study of the clinical days. For the community breastfeeding assessment, learners return to their home communities and research breastfeeding rates and support by reaching out to local hospitals, the WIC program, and healthcare provider offices. The goal of this assignment is to encourage the learner to start building relationships with others in their community who support breastfeeding.

Comprehension of the breastfeeding material is assessed through the learner's completion of 15 competency-based application questions. In addition, the learners develop a World Breastfeeding Week activity and complete a report, which encourages them to apply the information learned to an activity for their place of employment. If a learner works in a hospital setting and is able to complete a hospital profile, they may complete the profile in lieu of the World Breastfeeding Week activity. The reference review requires that students review and critique five lactation references or resources used in their specific work location. Each learner is required to complete a case study related to the clinical day experience and reflect on the status of their breastfeeding skills and ways to improve.

The NCLETP is accredited by the Lactation Education Accreditation and Approval Review Committee and is awarded 69 IBLCE Continuing Education Recognition Points (CERPS; 64 L CERPS, 4.25 R CERPS, 0.750 E CERPS). This accreditation is for programs which have been in existence for more than 1 year, offers a minimum of 45 contact hours, and covers areas in the IBLCE Exam Blueprint, the primary faculty have been IBCLCs for more than 5 years, and the faculty that teach more than $10 \%$ of the course are IBCLC certified. As an alternative, the program is approved for 69 contact hours from Northwest AHEC for nurses, social workers, peer counselors, dietitians, and educators. The credit hours provided assist health professionals to maintain their nursing or dietitian certification and to accrue the lactation continuing education to sit for the IBLCE certification exam. 


\section{Impact}

To date, the NCLETP has reached 1,500 healthcare staff members who have come from all 100 counties in North Carolina. Most participants have been either nutritionists/dietitians, nurses, or breastfeeding peer counselors. Other providers attending include WIC staff, IBCLCs, educators, nurse practitioners, social workers, certified medical assistants/certified nursing assistants, physicians, medical students, parenting counselors, doulas, and La Leche League members.

One of the initial goals of this program was to target care providers in health departments and those working with WIC. The program has expanded and now includes staff working in other locations as well. Currently, about half of the learners report working in hospitals. With the change in federal policy with the Affordable Care Act requiring all healthcare providers to provide breastfeeding support to their patients, more staff from healthcare provider offices are now attending the NCLETP. Almost all of the learners enrolled have been residents of North Carolina. However, providers from Virginia, South Carolina, Georgia, and Mississippi have participated through the years.

Most learners work in either public health departments or hospitals. Others report places of employment as being rural and urban physician offices, primary care clinics, schools of nursing, solo practice, and schools. In addition, a few college students working on a graduate degree have attended the program through its 20 years of existence (Ward \& Byrne, 2011).

\section{Outcomes}

The total number of clients served by NCLETP-trained providers is approximately 250,000 per year. This number includes pregnant women seen in hospitals, health departments, physician offices, and clinics. This volume also reflects postpartum counseling by program participants during WIC visits, hospital stays, and home visits by nurses, social workers, and doulas.

The NCLETP is not intended to replace the IBCLC credential, and learners are informed about this important distinction on the first day of the program. Faculty members discuss the process of IBCLC credentialing and promote this as a potential goal. Participants are made aware of the ability to actually use the continuing education hours from the NCLETP as partial fulfillment toward the initial IBCLC designation. There is usually a vigorous discussion about this possibility among program participants, many of whom which to eventually become an IBCLC. Of note, $5 \%$ of total participants have become credentialed as IBCLCs. As a result of the NCLETP, there are 70 new IBCLCs throughout the state of North Carolina.

When the NCLETP started in 1996, approximately 26\% of the women enrolled on the WIC program initiated breastfeeding. The 2015 WIC data indicate that $72 \%$ of the women enrolled are initiating breastfeeding. Over the past 20 years, there has been a $177 \%$ increase in the number of women enrolled in the WIC program who initiate breastfeeding; both the NCLETP, increased trained staff members, and peer counselor programs have contributed to this rise. The 2007 Breastfeeding Report Card Data from the Centers for Disease Control and Prevention (CDC) indicates that $72 \%$ of the women in North Carolina had ever breastfed their infants. From 2007 to 2014, there was a 7\% increase in the number of women ever breastfeeding with $77 \%$ of the women in North Carolina initiating breastfeeding.

In examining the CDC Breastfeeding Report Card Data from 2007 to 2014, North Carolina has seen increases in both breastfeeding duration and exclusivity. For North Carolina, the 6-month duration increased by $41 \%$, and the 12 -month duration increased by $34 \%$ during the 7-year period. The greatest improvement for North Carolina has been in the exclusivity rate with an increase of $85 \%$ for 3 months exclusivity and 195\% for breastfeeding exclusivity at 6 months. Data from the North Carolina WIC program indicates significant increases in breastfeeding duration among this population. There was a $157 \%$ increase in the number of women breastfeeding at 12 months from 1993 to 2011, going from $7 \%$ in 1993 to $18 \%$ in 2011. For the WIC population, the greatest increases were seen in duration at 6 weeks ( $83 \%$ increase) and 6 months ( $108 \%$ increase).

\section{Evaluation}

An evaluation is given to program participants 1 week after completion, allowing them to rate the entire program using a Likert scale. Ninety-five percent of participants either strongly agreed or agreed that program faculty members were knowledgeable regarding subject matter. In addition, 95\% of the responses either strongly agreed or agreed that the program was presented in a fair/unbiased manner.

Meeting the NCLETP program objectives is also evaluated. One hundred percent of the respondents either strongly agreed or agreed that they were able to 
describe the advantages of human milk for infant feeding. Another 95\% either strongly agreed or agreed that they were able to discuss factors contributing to breastfeeding success. Also, 90\% either strongly agreed or agreed that they could troubleshoot common problems, such as refusal to nurse.

Other program objectives are also evaluated: $95 \%$ of respondents strongly agreed or agreed that they could both explain strategies to help a teen mother breastfeed and develop one goal to improve breastfeeding support in their workplace. Another 90\% strongly agreed or agreed that they could describe management of special situations, such as diabetes.

In addition, $100 \%$ of participants strongly agreed or agreed that they were able to recognize quality resources for best breastfeeding evidence-based practice. Eightyfive percent either strongly agreed or agreed that they could plan to formulate a standard protocol for use in their hospital or health department.

\section{Participant Evaluation Comments}

Participants are asked for comments as a part of the initial evaluation process, and they have included the following:

- I will use this training to educate more young families about breastfeeding during home visits.

- I can offer lactation support in our private practice pediatrician office.

- I will be more encouraging of my clients to breastfeed.

- I'm more culturally aware of breastfeeding concerns and patterns.

- I am more aware of evidence-based practice now.

- I plan to visit local hospital to assess their breastfeeding practices.

- I am more confident troubleshooting instead of always referring.

- I will really stress breastfeeding importance to teen moms.

- Hospital and health department clinical experiences were excellent.

- Discovering local breastfeeding resources and contacts was valuable.
- The discussion of state and national breastfeeding policies was helpful.

Long-term evaluation comments, conducted 6-12 months after NCLETP completion, revealed the following:

- I am counseling moms with positioning and latching on.

- I know how to support and counsel moms with premature babies.

- I learned a lot about breastfeeding twins or tripletsthis has been helpful to me.

- We care for a lot of diabetic moms, and I now know there could be a delay in their milk supply.

- Hands-on experience at the hospital increased my self-confidence with new breastfeeding moms.

- I am now facilitating our breastfeeding mother's support group.

- I am a much more effective listener with clients and better able to assist with breastfeeding issues.

- We have started lactation home visits, knowing how important it is to correct problems early.

\section{Future Directions}

The NCLETP continues into 2017, with 7-day sessions held each spring and fall. The cost of the program is affordable for participants, with many faculty members donating their professional expertise. Workshop evaluations are examined on a continual basis for needed changes in topics, delivery methods, or faculty members.

As a result of evaluation comments, an annual spring continuing education conference, "Breastfeeding Forum: Practices for the Future" is being held to keep trained lactation educators up to date with recent advances in the field. This conference is administered through Northwest AHEC, is held at Novant Health Forsyth Medical Center, and is open to all providers in the state. Continued support and participation from all the associated partners is critical to continuation of the NCLETP in the future.

\section{References}

Academy of Breastfeeding Medicine. (2011). Educational objectives and skills for the physician with respect to breastfeeding. Breastfeeding Medicine, 6(2), 99-105. http://dx.doi.org/10.1089/ bfm.2011.9994 
Howett, M., \& Lauwers, J. (2013). The standardization of lactation education to improve professionalism and patient care. Journal of Human Lactation, 29(4), 449-451. http://dx.doi .org/10.1177/0890334413477917

Maskey, C. L. (2008). The coordination of clinical and didactic learning experiences to improve critical-thinking skills and academic performance. Teaching and Learning in Nursing, 3, 11-15. http://dx.doi.org/10.1016/j.teln.2007.07.011
Ward, K. N., \& Byrne, J. P. (2011). A critical review of the impact of continuing breastfeeding education provided to nurses and midwives. Journal of Human Lactation, 27(4), 381-393. http:// dx.doi.org/10.1177/0890334411411052

Watkins, A. L., \& Dodgson, J. E. (2010). Breastfeeding educational interventions for health professionals: A synthesis of intervention studies. Journal for Specialists in Pediatric Nursing, 15(3), 223-232. http://dx.doi.org/10.1111/j.1744-6155.2010.00240.x

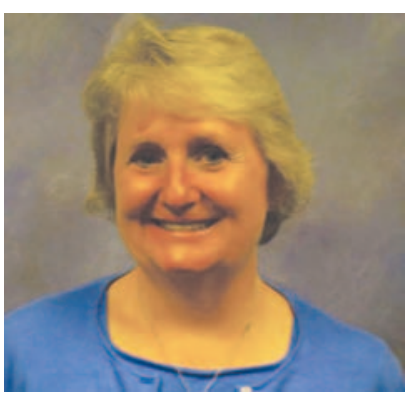

Georganna Cogburn, MSHE, RD, LDN, IBCLC, RLC, is the Region 1 lactation trainer for the western 16 counties of North Carolina from the Mountain Area Health Education Center. She was a public health nutritionist for 29 years with the WIC program where she assisted women with breastfeeding their infants. Georganna has been an IBCLC since 1992 and a registered dietitian since 1984. She has been the Region 1 breastfeeding coordinator since 2005 and in that capacity has trained breastfeeding peer counselors and health department staff throughout Western North Carolina. Since 1996, she has been on the faculty of the NCLETP which is a program offered in conjunction with the North Carolina Department of Health and Human Services, Northwest Area Health Education Center, and Novant Health. She is also an adjunct professor at Western Carolina University in the School of Health and Human Sciences.

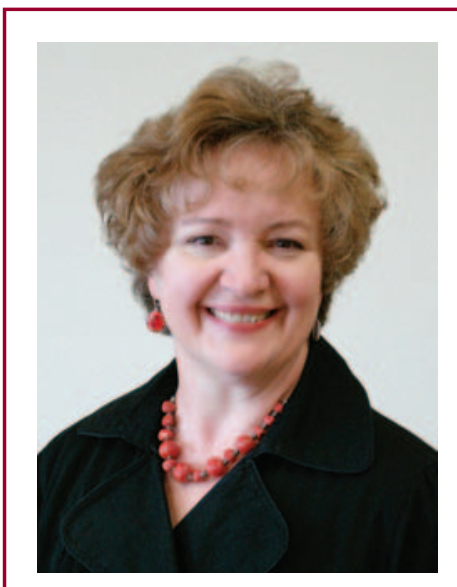

Mona Brown Ketner, RN, MSN, C-EFM, graduated from the University of North Carolina (UNC)-Chapel Hill in 1978 with a bachelor of science in nursing degree and from UNC-Greensboro in 1983 with a master of science in nursing. She has worked in various hospitals throughout the state of North Carolina-including Rex Hospital in Raleigh, Forsyth Memorial Hospital in Winston-Salem, and Hoots Memorial Hospital in her hometown of Yadkinville. Mona came to Bowman Gray School of Medicine in 1990, providing home visits and telephone counseling to women in an effort to help prevent preterm births. Afterward, she served as the nurse counselor with Breath of Fresh Air, a telephone counseling program funded by the March of Dimes, to assist women in Forsyth County with quitting tobacco use. Mona then moved into the role of perinatal outreach educator at Wake Forest University School of Medicine for 15 years, where she taught multiple continuing education programs in northwest North Carolina-including fetal monitoring, telephone triage, postpartum assessment, smoking cessation, and depression in women. She holds a certificate in electronic fetal monitoring from the National Certification Corporation. Mona is currently a Nurse Educator with Northwest Area Health Education Center at Wake Forest School of Medicine, teaching continuing education programs to care providers within a 17-county region. 